\title{
Long-term clinical outcome following lower limb arterial angioplasty
}

\author{
G Morris-Stiff', M Moawad', N Appleton¹, G Davies², E Hicks², C Davies², MH Lewis ${ }^{1}$
}

Departments of Surgery ${ }^{1}$ and Radiology ${ }^{2}$, Royal Glamorgan Hospital, Ynysmaerdy, Llantrisant, UK

\begin{abstract}
INTRODUCTION The aim of this study was to determine the clinical outcome of lower limb arterial angioplasty in a busy district general hospital practice.

PATIENTS AND METHODS All angioplasties performed from January 1999 to December 2004 were identified and data collected included cardiovascular risk factors, indications for and complications of angioplasty, limb salvage and patient survival rates, and clinically significant re-stenoses.

RESULTS 471 interventions were performed in 385 patients (231 men, 154 women). The median age was 67.9 years (range: 39-93 years). Indications for angioplasty were critical ischaemia ( $n=247,52 \%)$ and lifestyle-limiting intermittent claudication ( $n=224,48 \%)$. Stenotic lesions accounted for 378 (80\%) cases and occlusion for 93 (20\%). Radiological success was obtained in 417 (88.5\%), improving to $93.6 \%$ if only those in whom access was achieved were included. Post-angioplasty complications were observed in $42 / 471(9.1 \%)$ of interventions. The actual patient survival at 1 , 2 , and 3 years was $87.4 \%$, $85.1 \%$ and $83.2 \%$ respectively. Indication for angioplasty and the number of lesions present were identified as risk factors for outcome on multivariate analysis. The cumulative post-angioplasty patency rates at 1,2 and 3 years were $86.0 \%, 83.1 \%$ and $81.6 \%$ respectively. The only factor associated with patency was the mode of presentation.

CONCLUSIONS Angioplasty for lower limb peripheral vascular disease can be performed safely and efficaciously with a high technical success rate and a low complication rate. The patient survival and post-angioplasty patency data reflect the progressive and multi-site nature of the underlying disease process.
\end{abstract}

\section{KEYWORDS}

Angioplasty - Peripheral vascular disease - Ischaemia - Clinical outcome - Survival

Accepted 12 January 2010

\section{CORRESPONDENCE TO}

Michael H Lewis, Consultant Vascular Surgeon, Royal Glamorgan Hospital, Ynysmaerdy, Llantrisant CF72 8XR, UK T: +44 (0)1443 443 838; F: +44 (0)1443 443 224; E: mike.lewis@pr-tr.wales.nhs.uk

The concept of angioplasty, first described by Dotter and Jenkins in $1964,{ }^{1}$ has now become the treatment of choice for arterial stenoses in most anatomical locations, with level I evidence ${ }^{2}$ for the treatment of both lifestyle-limiting intermittent claudication and critical ischaemia, and the establishment of treatment guidelines for each type of lesion., ${ }^{2,5}$

In an overview of the results of lower limb angioplasty, Davies et al reported initial radiological success rates of 90$95 \%$ for single stenotic lesions and $80-95 \%$ for occlusions of the femoropopliteal segment using a standard antegrade femoral approach ${ }^{4}$ with patency rates of $71 \%, 59 \%$, and $53 \%$ at one, three and five years respectively.

A number of factors have been shown to compromise patency rates: lesion characteristics - location, nature of lesion (occlusion vs stenosis), length, number of lesions, multilevel disease, adequate run-off; demographics - gender, diabetes mellitus; clinical - indication (intermittent claudication vs critical ischaemia), recurrent lesions; and procedure-related - vessel dissection, radiological response..$^{5-9}$

There are a number of procedure-related complications associated with angioplasty with a mean overall rate of $10 \%$ reported by Davies et al..$^{5}$ The prevalence of recog- nised complications of angioplasty was summarised in the American Heart Association (AHA) guidelines and includes: severe bleeding $3.4 \%$; false aneurysm $0.5 \%$; arteriovenous fistula $0.1 \%$; thrombosis $3.2 \%$; rupture $0.3 \%$; dissection $0.4 \%$; embolisation $2.3 \%$; emergency operation $2 \%$; limb loss $0.2 \%$; and mortality $0.2 \% .^{10}$

The main difficulty in interpreting much of the data in the literature is that it represents the experiences obtained in large-volume tertiary referral centres and thus there is some question regarding its applicability to non-specialist practice. With the proliferation of angioplasty and a recognition of its benefits over surgery in many patients, there has been a greatly increased demand for the service, leading to the development of treatment programmes outside the larger tertiary centres.

The aim of this study was to report outcomes of a lower limb angioplasty programme from a non-teaching hospital setting.

\section{Patients and Methods}

All patients undergoing lower limb angioplasty during the period January 1999 to December 2004, were identified from 


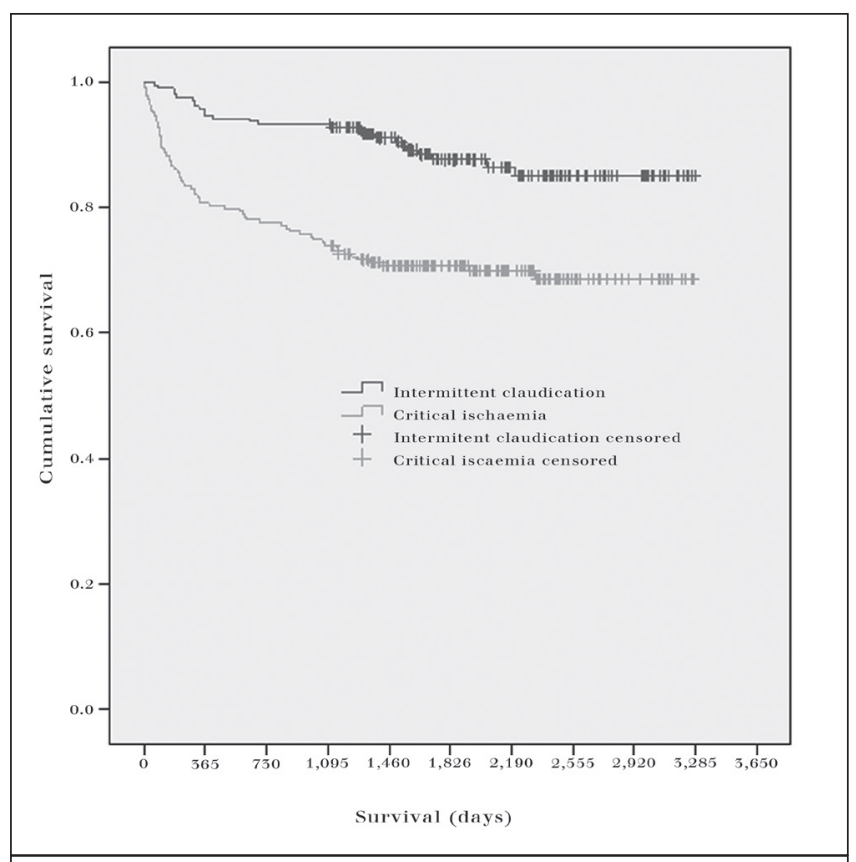

Figure 1 Patient survival according to clinical indication for operation

Table 1 Risk factors for cardiovascular disease in patients undergoing angioplasty

\begin{tabular}{|lll|}
\hline Risk factors & Yes & No \\
\hline Smoking & & $80(17 \%)$ \\
$\quad$ Smoker & $220(46.7 \%)$ & \\
$\quad$ Ex-smoker & $171(36.3 \%)$ & \\
Diabetes & $143(30 \%)$ & $328(70 \%)$ \\
Hypertension & $238(50.5 \%)$ & $233(49.5 \%)$ \\
Hyperlipidaemia & $165(35 \%)$ & $306(65 \%)$ \\
Ischaemic heart & $159(33.8 \%)$ & $312(66.2 \%)$ \\
disease & $97(20.6 \%)$ & $374(79.4 \%)$ \\
Stroke & $28(5.9 \%)$ & $443(94.1 \%)$ \\
Renal failure & & \\
\hline
\end{tabular}

a prospectively maintained radiology database. Those undergoing primary stenting procedures were not included in this analysis.

Data collected from patient records included: indications for angioplasty; cardiovascular risk factors; location, number and nature of lesions; complications of angioplasty; limb salvage rates; and rates of clinically significant re-stenosis.

Data analysis was performed using SPSS $^{\circledR}$ v15.0 (SPSS Inc, Chicago, Illinois, US). Statistical significance was assessed at the 5\% level. The Kaplan-Meier method was used to assess the overall survival and post-angioplasty patency rates. A univariate analysis was performed to assess for a significant difference in factors believed to be of importance in relation to overall survival and post-angioplasty patency.

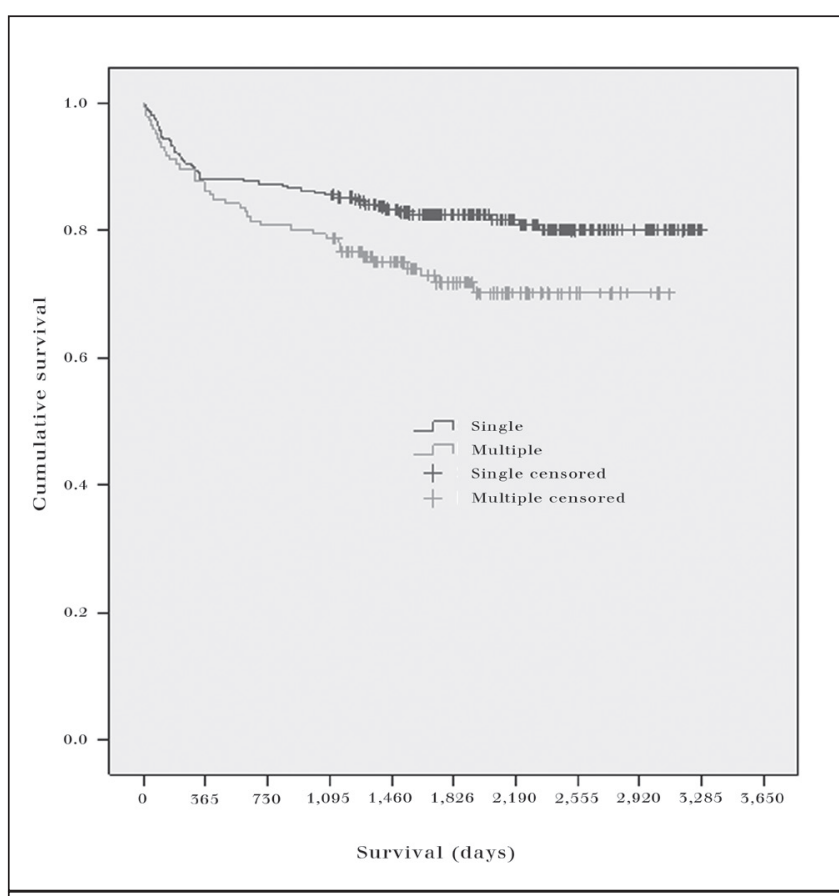

Figure 2 Patient survival according to number of lesions present

\section{Table 2 Indications for angioplasty}

\begin{tabular}{ll}
\hline Risk factors & Number \\
\hline Indication for angioplasty & \\
Critical ischaemia & $247(52 \%)$ \\
Lifestyle-limiting intermittent & $224(48 \%)$ \\
claudication & \\
Type of lesion & \\
Stenosis & $378(80 \%)$ \\
Occlusion & $93(20 \%)$ \\
Number of stenoses & $259(68.5 \%)$ \\
Single & $119(31.5 \%)$ \\
Multiple & \\
Number of occlusions & $59(63.4 \%)$ \\
Single & $34(36.6 \%)$ \\
Multiple &
\end{tabular}

A multivariate analysis was then performed by Cox regression (stepwise forward model) on those variables identified during univariate analysis.

\section{Results}

A total of 471 angioplasties were attempted. The majority of procedures were performed in men $(n=317,67.3 \%)$ and the mean patient age was 67.9 years (range: $39-93$ years).

The cardiovascular risk factor profiles are summarised in Table 1. As a result of patient demographics and co-morbidities, the vast majority of procedures $(n=457,97 \%)$ were 


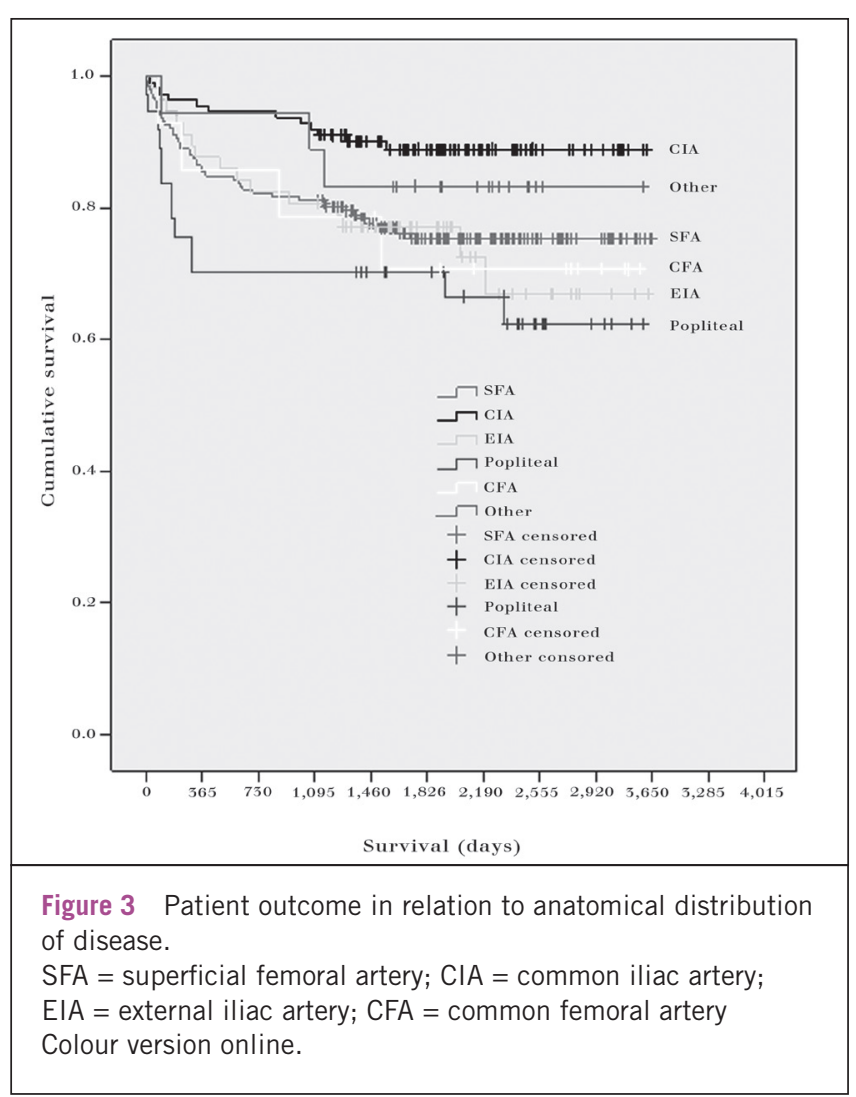

performed as inpatient procedures.

The indications for angioplasty are summarised in Table 2 and the distribution of lesions subjected to angioplasty in Table 3.

A good radiographic result was observed in 417 (88.5\%) cases. The most common reason for failure was abandonment due to inadequate access $(n=29)$. In the remaining 25 patients, angioplasty was performed but the radiological result was deemed inadequate. If only those in whom access was possible are included, the success rate improves to $363 / 388(93.6 \%)$.

The complication rate was $43 / 471(9.1 \%)$ (Table 4$)$. However, it fell to $4.5 \%$ when only those considered by the $\mathrm{AHA}^{2}$ were included. None of the patients required emergency operation, intervention or a blood transfusion.

There were eight $(1.7 \%)$ deaths within thirty days of angioplasty. The patients in this group had a mean age of 77 years, all had critical ischaemia with ulceration as the indication for angioplasty and a history of cardiovascular disease. None of the patients experienced a complication of angioplasty and all died following a myocardial event.

Immediate radiological success rates for single and multiple stenoses were $92.1 \%$ and $91.4 \%$ respectively, whereas the initial success following angioplasty of single and multiple occlusions was $80.8 \%$ and $71.9 \%$.

Fifty-eight patients underwent bypass surgery $(n=47)$ or stenting $(n=11)$ following angioplasty. Twenty-nine patients had a procedure less than three months after surgery, six of which had experienced an angioplasty-related complication (dissection $[n=2]$; extravasation $[n=1]$; perforation $[n=1]$;

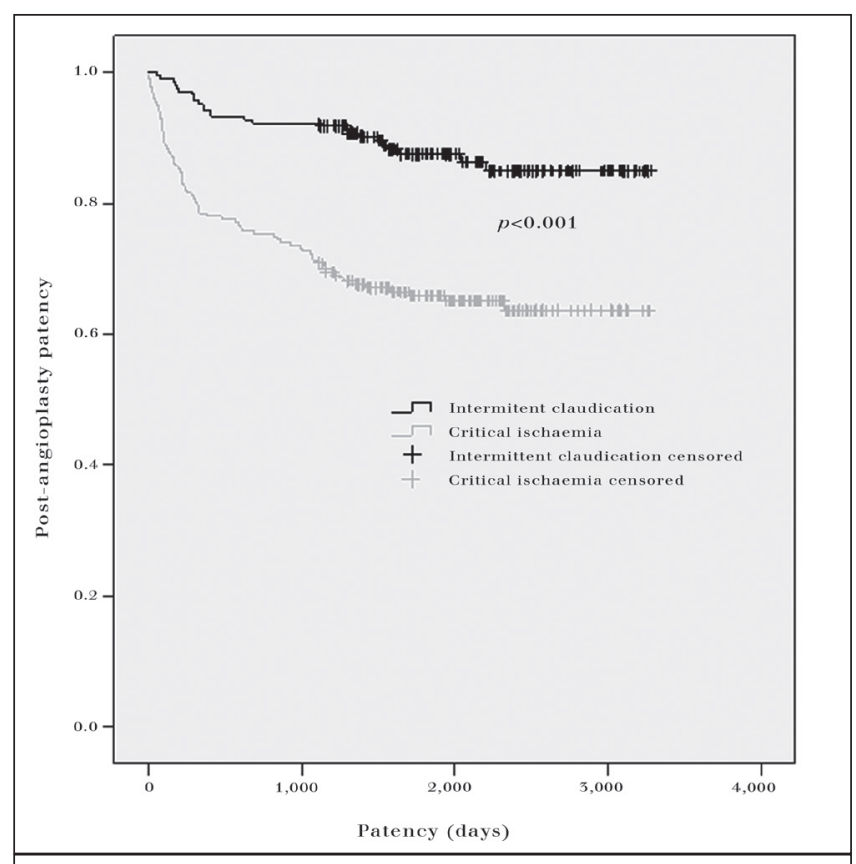

Figure 4 Post-angioplasty patency according to clinical indication for operation

haematoma $[n=1]$; sub-intimal dissection $[n=1]$.) In the remaining 23 patients, the angioplasty was performed as part of a management plan to dilate a stenosis in order to obtain either improved inflow or outflow prior to a surgical bypass.

In 29 cases where surgery was performed at an interval of more than 3 months, the mean interval between angioplasty and bypass surgery/stenting was 313 days (range: 91-1,785 days). In 11 of 29 cases, stenoses were within the same arterial segment and regarded as recurrences, whereas the remaining 18 lesions were regarded as de novo, occurring in a different segment of artery or contralateral limb.

Eight patients underwent amputation following angioplasty. All of these initially presented with critical ischaemia and ulceration. Three amputations were performed within one month of angioplasty (18, 24 and 27 days), with angioplasty having been performed in order to optimise blood flow to the distal limb and maximise chances of stump healing. In the remaining four cases, amputation was performed at an extended interval (238, 253, 399 and 407 days) and in each case angioplasty had improved blood flow to the distal limb but was inadequate to provide healing.

One hundred and eight patients had angioplasty performed for leg ulcers. In 95 of these cases, angioplasty was the sole treatment modality and $49(51.6 \%)$ of these healed completely.

Mean patient follow up from angioplasty to surgical intervention, patient death or end of study was 28.3 months (range: $0-80$ months). To more accurately assess the role of angioplasty, procedures performed prior to elective surgery were excluded, as were those performed following previous surgery or amputation, leaving 430 angioplasties for analy- 


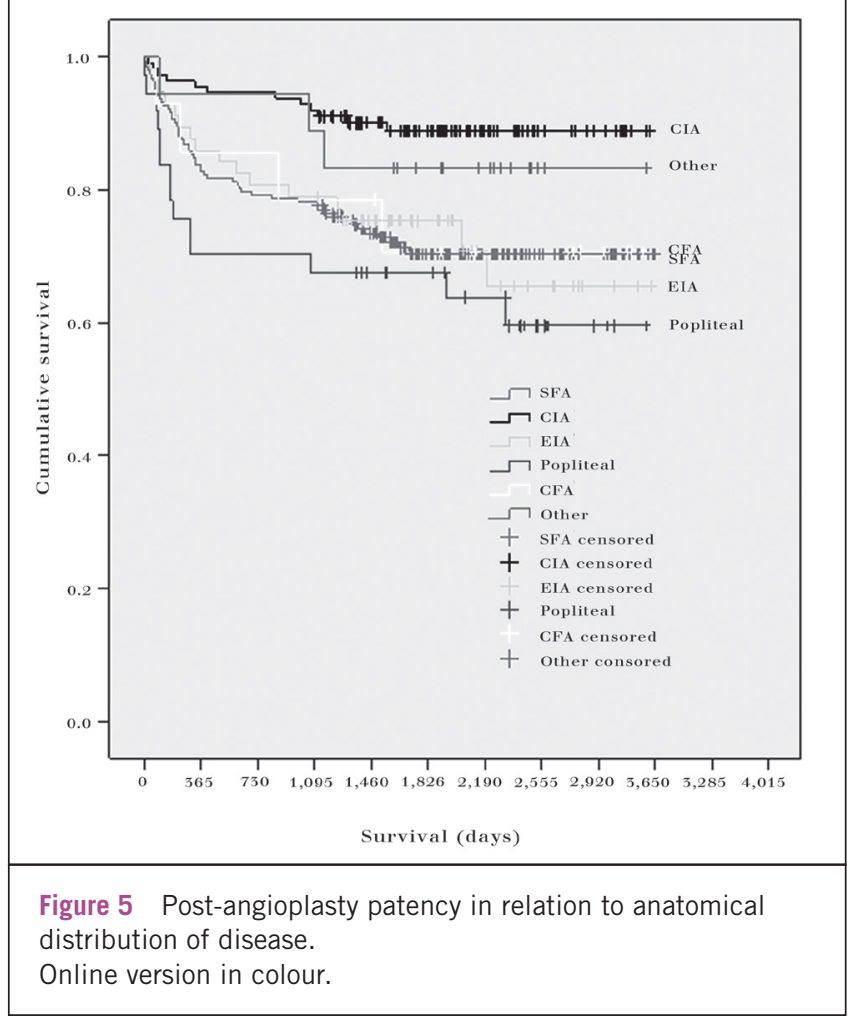

sis.

\section{Patient survival}

Univariate analysis revealed a significant difference in survival based on the indication for angioplasty (Fig 1) (critical ischaemia vs intermittent claudication; $p<0.001$ ) and number of lesions present (Fig 2) (multiple vs single; $p=0.02$ ). The actual overall patient survival at 1,2 and 3 years was $87.4 \%, 85.1 \%$ and $83.2 \%$ respectively, while the 1 , 2 and 3-year overall survival rates for patients with intermittent claudication were $94.7 \%, 93.2 \%$ and $93.2 \%$. For critical ischaemia the corresponding rates were $80.7 \%, 77.6 \%$ and $74.4 \%$. In comparing single to multiple lesions, the overall survival rates at 1 year were $88 \%$ vs $85.6 \%$, at 2 years they were $87.3 \%$ vs $80.1 \%$ and at 3 years $85.6 \%$ vs $78.8 \%$. There was no difference in outcome when comparing the nature of the lesion present $(p=0.416)$, radiological outcome of angioplasty $(p=0.696)$ or development of complications $(p=0.938)$.

Subsequent multivariate analysis revealed that presentation (risk ratio $=2.89 ; 95 \%$ confidence interval $=1.82$ 4.58 ) and number of lesions (risk ratio $=1.60$; $95 \%$ confidence interval $=1.06-2.44$ ) remained significant predictors for overall survival.

There was a wide distribution in outcome according to the location of the main lesion (Fig 3) with common iliac artery angioplasty superior to: popliteal ( $p \leq 0.001)$; superficial femoral artery $(p=0.006)$; external iliac artery $(p=0.008)$; and common femoral artery $(p=0.05)$.

\section{Angioplasty patency}

The actual graft patency at one, two and three years was
Table 3 Distribution of lesions requiring angioplasty $(n=557)$

\begin{tabular}{|ll|}
\hline Site of the lesion & Number of cases \\
\hline Superficial femoral artery & 217 \\
Common iliac artery & 127 \\
External iliac artery & 81 \\
Popliteal artery & 80 \\
Common femoral artery & 25 \\
Other sites & 29 \\
\hline
\end{tabular}

Table 4 Complications experienced following angioplasty $(n=43)$

\begin{tabular}{ll}
\hline Complication & Number \\
\hline Small haematoma & $12(2.5 \%)$ \\
Dissection & $9(1.9 \%)$ \\
Spasm & $7(1.5 \%)$ \\
Sub-intimal dissection & $4(0.8 \%)$ \\
Thrombosis & $4(0.8 \%)$ \\
Pain & $3(0.6 \%)$ \\
Embolisation & $2(0.4 \%)$ \\
Extravasation & $1(0.2 \%)$ \\
Perforation & $1(0.2 \%)$ \\
\hline
\end{tabular}

$86.0 \%, 83.1 \%$ and $81.6 \%$ respectively, with the indication for angioplasty (Fig 4) (critical ischaemia vs intermittent claudication; $p<0.001$ ) being the only significant factor. The one, two and three-year overall survival rates for patients with intermittent claudication were $94.2 \%, 92.3 \%$ and $92.3 \%$, whereas for critical ischaemia the corresponding rates were $78.5 \%, 75.3 \%$ and $71.7 \%$.

There was no difference in outcome when comparing the number of lesions $(p=0.064)$, the nature of the lesion present $(p=0.437)$, radiological outcome of angioplasty $(p=0.955)$ or development of complications $(p=0.761)$.

The distribution of outcome in relation to the location of the main lesion is shown in Figure 5 and is similar to that seen for overall survival. The patency rates were highest in those undergoing angioplasty of the common iliac artery and worst for patients with infragenicular stenoses $(p \leq 0.001)$.

\section{Symptomatic outcome}

The majority ( $n=262,60.9 \%$ ) of patients reported that their claudication distances improved, with 123 (28.6\%) patients experiencing no change and $15(3.5 \%)$ reporting a deterioration. Data were absent for 30 patients $(7.0 \%)$. When the data were re-examined including only those with a successful radiological result, the improvement rate increased to $69.6 \%$ and the 'no improvement' rate fell to $3.7 \%$, leaving $26.7 \%$ unchanged. 


\section{Discussion}

The primary finding of this study is that angioplasty can be performed with acceptable periprocedural morbidity and good long-term outcome in a non-tertiary centre setting.

The proportion of patients undergoing angioplasty for critical ischaemia in the current study (52\%) was greater than in reported meta-analyses of angioplasty for iliac ${ }^{11}$ and femoropopliteal disease. ${ }^{12,13}$

The majority of angioplasties were performed for stenotic lesions, this being the case in most of the published literature.$^{11-14}$ However, almost a third of those with stenoses had more than one lesion treated by angioplasty. Furthermore, even though occlusive lesions accounted for only $20 \%$ of cases, a third had more than one occlusion.

Davies et al indicated that the overall complication rate of angioplasty should be less than $10 \%$, while in recent meta-analyses only major complications were included. ${ }^{11-14}$ The prevalence of major complications in the current series at $4.5 \%$ is in keeping with these meta-analyses where rates were $4.3-8.1 \%$.

The 30-day mortality appears high at $1.7 \%$. However, the proportions of critically ischaemic patients was higher and the major complication rates favourable, suggesting patient rather than technical factors as the cause of the increased mortality. Moreover, demographic and co-morbidity data were missing from the other series, thus adding further obstacles to a fair comparison of the series.

The fact that overall survival and patency rates were very similar and that the number of patients requiring bypass surgery or amputation were small suggests that the main factor determining outcome is the extent of the atherosclerotic cardiovascular disease. It is well recognised that patients with peripheral vascular disease have a high prevalence of cardiovascular disease and that the majority of claudicants (in excess of $80 \%$ ) die of cardiovascular causes. ${ }^{15-19}$ Indeed, the mortality of individuals with claudication is four times greater than an otherwise comparable population..$^{20}$ This also implies that patients with peripheral vascular disease, even in the absence of symptomatic cardiovascular disease, require treatment of cardiovascular risk factors. ${ }^{21}$

The patency rates in this series were superior to those in the meta-analyses for iliac ${ }^{11}$ and femoropopliteal disease. ${ }^{12,13}$ In the current series, the only factor associated with a poorer post-angioplasty patency was the indication for angioplasty, with critical ischaemia being associated with a poorer outcome.

Although a formal quality-of-life assessment tool was not used, $70 \%$ undergoing a technically successful procedure reported a significantly improved claudication distance following angioplasty. There are limited published data looking at the quality of life following angioplasty for lower limb vascular disease but the data that do exist suggest an improved quality of life following angioplasty. ${ }^{22,23}$

\section{Conclusions}

This study has demonstrated that angioplasty for peripheral vascular disease may be performed safely and efficaciously in a non-tertiary referral centre setting. The results of angioplasty in this series are comparable to those obtained from larger specialist vascular units in terms of complication rates and graft patency, with low rates of recurrence and a minimal requirement for further treatment (be it stenting, surgical bypass or amputation). Furthermore, our longterm survival appears to be better than expected and this may be related to our careful follow up of a local population.

\section{References}

1. Dotter CT, Judkins MP. Transluminal treatment of arteriosclerotic obstruction. Circulation 1964; 30: 654-670.

2. Hirsch AT, Haskal ZJ, Hertzer NR et al. ACC/AHA 2005 guidelines for the management of patients with peripheral arterial disease (lower extremity, renal, mesenteric, and abdominal aortic): executive summary. J Am Coll Cardiol 2006; 47: 1,239-1,312.

3. Norgren L, Hiatt WR, Dormandy JA et al. Inter-society consensus for the management of peripheral arterial disease (TASC II). J Vasc Surg 2007; 45 (Suppl): S5-S67.

4. Davies MG, Waldman DL, Pearson TA. Comprehensive endovascular therapy for femoropopliteal arterial atherosclerotic occlusive disease. J Am Coll Surg 2005; 201: 275-296.

5. Capek P, McLean GK, Berkowitz HD. Femoropopliteal angioplasty. Factors influencing long-term success. Circulation 1991; 83(2 Suppl): 70-80.

6. Johnston KW. Femoral and popliteal arteries: reanalysis of results of balloon angioplasty. Radiology 1992; 183: 767-771.

7. Hunink MG, Donaldson MC, Meyerovitz MF et al. Risks and benefits of femoropopliteal percutaneous balloon angioplasty. J Vasc Surg 1993; 17: 183-192.

8. Becquemin JP, Cavillon A, Haiduc F. Surgical transluminal femoropopliteal angioplasty: multivariate analysis outcome. J Vasc Surg 1994; 19: 495-502.

9. Clark TW, Groffsky JL, Soulen MC. Predictors of long-term patency after femoropopliteal angioplasty: results from the STAR registry. J Vasc Interv Radiol 2001; 12: 923-933.

10. Pentecost MJ, Criqui MH, Dorros G et al. Guidelines for peripheral percutaneous transluminal angioplasty of the aorta and lower extremity vessels. Circulation 1994; 89: 511-531.

11. Bosch JL, Hunink MG. Meta-analysis of the results of percutaneous transluminal angioplasty and stent placement for aortoiliac occlusive disease. Radiology 1997; 204: 87-96.

12. Hunink MG, Wong JB, Donaldson MC et al. Patency results of percutaneous and surgical revascularization for femoropopliteal arterial disease. Med Decis Making 1994; 14: 71-81.

13. Hunink MG, Wong JB, Donaldson MC et al. Revascularization for femoropopliteal disease. A decision and cost-effectiveness analysis. JAMA 1995; 274: 165-171.

14. Nicoloff AD, Taylor LM Jr, McLafferty RB et al. Patient recovery after infrainguinal bypass grafting for limb salvage. J Vasc Surg 1998; 27: 256-263.

15. Imparato AM, Kim GE, Davidson T, Crowley JG. Intermittent claudication: its natural course. Surgery 1975; 78: 795-799.

16. McAllister FF. The fate of patients with intermittent claudication managed nonoperatively. Am J Surg 1976; 132: 593-595.

17. Cronenwett JL, Warner KG, Zelenock GB et al. Intermittent claudication. Current results of non-operative management. Arch Surg 1984; 119: 430-436.

18. Leng GC, Fowkes FGR. The epidemiology of peripheral vascular disease. Vasc Med Rev 1993; 4: 5-18.

19. Walsh DB, Gilbertson JJ, Zwolak RM et al. The natural history of superficial femoral artery stenoses. J Vasc Surg 1991; 14: 299-304.

20. Smith GD, Shipley MJ, Rose G. Intermittent claudication, heart disease risk factors, and mortality. The Whitehall Study. Circulation 1990; 82: 1,9251,931 .

21. D'Souza J, Patel NN, Rocker M et al. Management of cardiovascular risk factors by primary care physicians in patients with peripheral arterial disease. Surgeon 2008; 6: 140-147.

22. Klevsgård R, Hallberg IR, Risberg B, Thomsen MB. The effects of successful intervention on quality of life in patients with varying degrees of lower-limb ischaemia. Eur J Vasc Endovasc Surg 2000; 19: 238-245.

23. Kalbaugh CA, Taylor SM, Blackhurst DW et al. One-year prospective quality-oflife outcomes in patients treated with angioplasty for symptomatic peripheral arterial disease. J Vasc Surg 2006; 44: 296-302. 